[0212-7199 (2005) 22: 8; pp 369-372] ANALES DE MEDICINA INTERNA Copyright (C) 2005 ARAN EDICIONES, S.L.

AN. MED. INTERNA (Madrid) Vol. 22, N. ${ }^{\circ} 8$, pp. 369-372, 2005

\section{Tratamiento domiciliario de la trombosis venosa profunda. Comparación de costes con la hospitalización convencional}

\author{
J. MONTES, L. GONZÁLEZ', L. AMADOR ${ }^{1}$, A. NOVO'1 , M. ENERO ${ }^{1}$, G. REY², \\ A. MEDIERO ${ }^{2}$
}

Servicios de Medicina Interna, ${ }^{1}$ Urgencias-HADO y ${ }^{2}$ Documentación Clínica. Hospital

Meixoeiro. Complejo Hospitalario Universitario. Vigo. Pontevedra

HOME VERSUS INPATIENT THERAPY FOR DEEP VENOUS THROMBOSIS. A COST-COMPARATIVE ANALYSIS

\section{RESUMEN}

Objetivos: Comparar los costes del tratamiento ambulatorio por una Unidad de Hospitalización a Domicilio (HADO) frente a la hospitalización convencional en el tratamiento agudo de la trombosis venosa profunda (TVP) y embolismo pulmonar (EP).

Métodos: Durante el año 2002 se trataron 21 pacientes con TVP en la Unidad de HADO. La mediana de edad fue de 81 años, 11 fueron mujeres $(52 \%)$ y, excepto uno, todos los pacientes presentaban importante comorbilidad. El diagnóstico se realizó en el hospital por ecografíadoppler. En 13 casos se realizó además una gammagrafía pulmonar, objetivándose EP concomitante en 7 pacientes. El tratamiento se realizó mediante heparinas de bajo peso molecular (HBPM) seguidas de anticoagulantes orales en 3 pacientes. No hubo complicaciones excepto un caso que requirió un ingreso breve debido al pobre control sintomático de la TVP y cuyos costes se imputaron a HADO. El estudio comparativo de costes se realizó con respecto a pacientes con TVP (grupo de diagnóstico relacionado, GDR 131) y EP ingresados (GDR: 78). El coste farmacológico para pacientes de HADO se calculó para 10 días.

Resultados: La estancia media hospitalaria de los pacientes ingresados fue de 8,1 días en TVP y 13,1 en TEP frente a 1 día en los pacientes en HADO. El ahorro de costes en HADO para el tratamiento agudo fue estimado en $1.680 €$ por paciente.

Conclusiones: El tratamiento ambulatorio mediante una unidad de HADO de pacientes con TVP (y TEP seleccionados) resultó una estrategia segura, eficaz y coste-efectiva.

PALABRAS CLAVE: Trombosis venosa profunda. Coste-efectividad. Heparinas de bajo peso molecular. Tratamiento ambulatorio.

\section{ABSTRACT}

Aims: To compare the home-care management of deep vein thromboses $(D V T)$ by a Home Care Unit $(H C U)$ respect to conventional inpatient treatment.

Methods: Twenty-one patients with a doppler-ecography diagnosis of DVT were managed by the HCU during 2002. In 7 out 13 a concomitant diagnosis of pulmonary embolism (PE) was made by lung scan. Median age was 81 years, $52 \%$ were women and all, except one case, showed severe medical concomitant conditions. All patients received low-weight molecular heparin, followed by oral anticoagulants in 3 patients. No patients died and only one was hopitalized briefly due to a poor thrombosis-related pain control. Costs of this patient were added to those of HCU. A comparison was made between ambulatory and hospitalary costs for EP and DVT. Pharmacological treatment costs were calculated for a 10-days period.

Results: The length of inhospital stay was 1 day for HCU vs. 8 days (DVT) and 13 days (EP). There was a estimated cost-saving of 1680 per patient.

Conclusions: The management of DVT in patients with serious conditions, can be accomplished safely and in a cost-saving manner by a Home Care Unit.

KEY WORDS: Deep vein thrombosis. Cost-effectiveness. Low-molecular weight heparin. Outpatient treatment.

Montes J, González L, Amador L, Novo A, Enero M, Rey G, Mediero A. Tratamiento domiciliario de la trombosis venosa profunda. Comparación de costes con la hospitalización convencional. An Med Interna (Madrid) 2005; 22: 369-372.

\section{INTRODUCCIÓN}

Se ha señalado recientemente que la enfermedad tromboembólica venosa (ETEV) constituye la cenicienta de las enfermedades cardiovasculares, si se compara con otras manifestaciones más estudiadas de trombosis arterial y presentadas clínicamente como infarto de miocardio (IAM), enfermedad cerebrovascular (ECV) o arteriopatía periférica (1). Sin embargo, la ETEV es una enfermedad frecuente, muchas veces infradiagnosticada y potencialmente mortal, debido a la posibilidad de fragmentación del trombo venoso con producción de un embolismo pulmonar (EP). Este constituye, de

Trabajo aceptado: 11 de mayo de 2005

Correspondencia: Julio Montes Santiago. Servicio de Medicina Interna. Hospital Meixoeiro. Complejo Hospitalario Universitario. 36200 Vigo, Pontevedra. e-mail: julio.montes.santiago@sergas.es 
hecho, la tercera causa de muerte intrahospitalaria tras el IAM y la ECV (2). Estudios poblacionales han estimado una incidencia anual de ETEV de 0,2\%, existiendo una acusada dependencia con la edad, doblándose su incidencia por cada década hasta alcanzar el 11\% a los 80 años (3-6). Se ha encontrado entre pacientes hospitalizados una incidencia 100 veces mayor que en ambulatorios (7). Prospecciones económicas realizadas para ciertos países occidentales (EE.UU., Japón, Francia, Alemania, Reino Unido, Italia y España) estiman en 1,2 millones los casos de ETEV anuales y en más de 52 millones los individuos susceptibles de profilaxis (8). Ello constituye un importante problema económico y así en USA se estiman unos costes para la ETEV de unos 1.500 millones de dólares/año (9). En nuestro país se estimó un coste para 1992 de 420,7 millones de $€$ (10).

La constatación de que las heparinas de bajo peso molecular (HBPM) son al menos tan efectivas y seguras como las heparinas no fraccionadas (HNF) en el tratamiento de la ETEV, llevaron en 1997 a la FDA, entre otros organismos, a permitir su utilización en el tratamiento de esta entidad. Esto constituyó la puerta de entrada para que muchos de estos pacientes pudieran beneficiarse de un tratamiento ambulatorio, con el consiguiente bienestar y ahorro de costes. Sin embargo, y por diversas circunstancias, muchos de estos pacientes aún siguen tratándose en régimen hospitalario. En nuestro país, son cada vez más frecuentes las comunicaciones que describen experiencias en el tratamiento ambulatorio con HBPM, pero prácticamente nulas las que analizan las ventajas económicas que pueden reportar. En este trabajo se refleja la experiencia de un año de una Unidad de Hospitalización a domicilio (HADO) en el tratamiento ambulatorio de pacientes con ETEV y se intenta una evaluación de sus costes en comparación con el tratamiento hospitalario convencional.

\section{MATERIAL Y MÉTODOS}

La Unidad de HADO del Hospital Meixoeiro en Vigo comenzó a funcionar a finales del año 2000. El objeto de este estudio lo constituyen los 21 pacientes remitidos con un diagnóstico de trombosis venosa profunda (TVP) para su tratamiento y seguimiento por dicha Unidad durante el año 2002. Todos tuvieron un diagnóstico confirmado de TVP proximal mediante ecografía-Doppler. En 13 se realizó además una gammagrafía pulmonar de ventilación/perfusión siendo de alta probabilidad para EP en 7 (58\%). Nuestro hospital dispone de tal exploración, incluyendo sábados. En cuanto al sexo 11 fueron mujeres (52\%). Presentaron una edad media de $77 \pm$ 13 años (mediana: 81, rango: 38-96). Dicha edad fue significativamente superior $(\mathrm{p}=0,03)$ para los pacientes de HADO que para los pacientes ingresados con un diagnóstico de ETEV en el Servicio de Medicina Interna (MI), y que también presentaron importantes patologías concomitantes [MI: 75 pacientes, edad media: $68 \pm 18$ años (mediana: 70, rango: 18 92); 29 EP, 28 EP+TVP, 18 TVP]. En MI hubo 47 mujeres (63\%) (NS, respecto a HADO). En cuanto a los factores predisponentes de trombosis estos fueron: a) inmovilización de diverso grado por enfermedad cerebrovascular o demencia avanzada: 8 casos; b) enfermedad neoplásica avanzada: 4 casos; c) enfermedades médicas previas graves: 6 casos (insuficiencia cardiaca crónica (2), cardiopatía isquémica (2), estenosis aórtica severa (1), EPOC (1), Glomerulonefritis (1)]; y d) cirugía previa (artroscopia): 1 caso. Este paciente fue el único sin procesos comórbidos importantes. Todos los pacientes fueron tratados con HBPM (10 casos con dalteparina, 11 casos con nadroparina). En 3 se utilizaron anticoagulantes orales concomitantes. El estudio comparativo de costes se realizó con respecto a los pacientes ingresados en el Hospital Meixoeiro en el año 2002 para un tratamiento durante la fase aguda. Se emplearon para ello los grupos de Diagnóstico Relacionados (GDR). Los costes y las características de los procesos considerados fueron: GDR 78 (embolismo pulmonar): coste total por proceso: $3.305,9 €$; estancia media: 13,1 días], GDR 131 (trastornos vasculares periféricos sin complicaciones): coste total por proceso: $1.772,6 €$; estancia media: 8,2 días]. Aunque los pacientes con trombosis venosa profunda quedaron agrupados bajo otros posibles GDRs (p. ej. 128, 131 y 543), se eligió el GDR 131 por ser en el que quedaron encuadrados el mayor número de pacientes en nuestro hospital. En la tabla I se detallan los costes considerados en los pacientes atendidos por HADO y facilitados por el Servicio de Contabilidad Analítica de nuestro hospital. Para el tratamiento farmacológico se utilizaron los precios de referencia para 10 días del Vademecum Internacional (Libro Rojo, Madrid, Media Medicom, 2002). Para el precio de las analíticas se utilizaron referencias publicadas (11). No hubo ningún fallecimiento durante los 10 días considerados. Un paciente con EP y TVP fue remitido al hospital por intensificación de la sintomatología inflamatoria en la extremidad inferior, siendo ingresado durante un día y vuelto a remitir a HADO. En este se repercutieron en los costes de HADO el equivalente a 3 días de estancia hospitalaria por EP. En el resto de pacientes no se observaron complicaciones significativas.

TABLA I

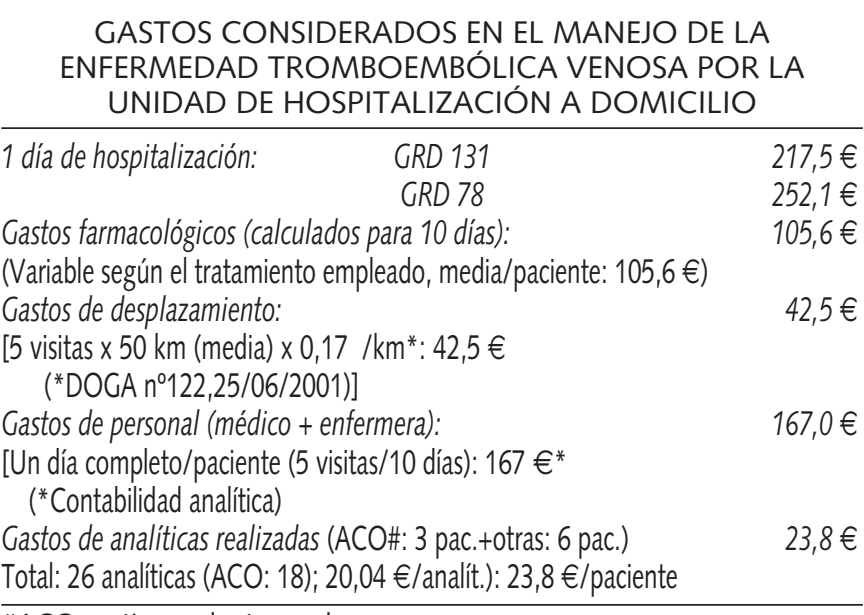

\#ACO: anticoagulantes orales

\section{RESULTADOS}

En la tabla II se consignan los costes totales de HADO y su comparación con los obtenidos en el supuesto de que dichos pacientes hubieran sido internados en una unidad de hospitalización convencional. La estancia en el Servicio de 
TABLA II

CÁLCULO DEL AHORRO DE COSTES EN HOSPITALIZACIÓN A DOMICILIO (LOS PACIENTES REMITIDOS A DICHA UNIDAD SÓLO PERMANECIERON UN DÍA EN EL SERVICIO DE URGENCIAS)

\begin{tabular}{lcc}
\hline & TVP & $E P$ \\
\hline Casos & 14 & 7 \\
Estancia media en pacientes ingresados (días) & 8,1 & 13,1 \\
Gastos hospitalarios (€) & 24.816 & 23.141 \\
Gastos hospitalarios totales $(€)$ & \multicolumn{2}{c}{47.958} \\
Coste real HADO* $(€):$ & \multicolumn{2}{c}{12.686} \\
Ahorro por paciente**: & \multicolumn{2}{c}{$1.680 €$}
\end{tabular}

*Incluyendo reingreso de un paciente con progresión de TVP

* * Gastos hospitalarios totales-coste real HADO/21 pacientes

Urgencias no superó un día comparada con 8 para la TVP y 13 para el EP. Se estimó un ahorro de $1.680 €$ por cada paciente y 10 días de tratamiento del episodio agudo. A pesar de la avanzada edad, las patologías graves subyacentes y que en un tercio de los pacientes se demostró un EP, no hubo complicaciones reseñables y sólo un paciente precisó de un corto periodo de hospitalización.

\section{DISCUSIÓN}

Existe hoy abrumadora evidencia procedente de estudios randomizados, metanálisis y revisiones sistemáticas de que las HBPM son al menos tan efectivas y seguras como las HNF en el tratamiento de la ETEV y que este puede realizarse ambulatoriamente en un gran porcentaje de pacientes (oscilante entre un 50 y 90\% según las series publicadas (12-19)]. Varios trabajos han demostrado también los beneficios en cuanto a aceptabilidad y calidad de vida obtenidas con dicho tratamiento $(20,21)$. En la revisión de muchos de estos estudios ambulatorios se demuestra también su coste-efectividad, apreciándose globalmente una reducción de costes en torno a los 1,600 \$ por paciente $(16,22)$.

Sin embargo, debido a la variabilidad de los modelos asistenciales, muchos pacientes siguen tratándose en régimen hospitalario. En nuestro país por ejemplo según datos de la Encuesta de Morbilidad Hospitalaria las tasas de hospitalización por trombosis se han mantenido estables en el periodo 1997-2001 (en torno a 35/100.000 habitantes, unos 14.000 casos hospitalizados/año) (23), aunque las cifras totales, si se extrapolan datos de otros países, podrían ser de 90.000 ETEV/año (65.000 TVP y 25.000 TEP) (10). Recientemente, se han estimado para España unos gastos mínimos de unos 20 millones de $€(0,12 \%$ del gasto en atención hospitalizada) para pacientes médicos hospitalizados con TEP (unos 7.000 pacientes/año) (7). Se trata de pacientes de avanzada edad pues 1 de cada 2 son $>75$ años. No obstante, y dejando aparte el caso paradigmático de Canadá -cerca del $90 \%$ de pacientes con ETEV tratados ambulatoriamente-, evidencias procedentes de nuestro entorno inmediato indican que con motivación $\mathrm{y}$ actuaciones protocolizadas los resultados son altamente mejorables. Por ejemplo, en un centro pudo aumentarse en 5 años la cifra de pacientes tratados de forma ambulatoria en un $50 \%$ (18). En nuestro país son cada vez son más frecuente las comunicaciones sobre tratamiento ambulatorio, procedentes a menudo de Unidades de HADO (24-26).

En este estudio hemos añadido una comparación entre los costes de HADO y la hospitalización convencional para el tratamiento agudo de pacientes con ETEV, obteniendo un ahorro para la primera de $1680 €$. Como ya ha sido notado para otras entidades como la insuficiencia cardiaca, las comparaciones de costes entre países pueden resultar difíciles, dado que, que debido a los distintos modelos asistenciales, el coste para el mismo proceso puede variar notablemente (27). Si bien nuestro estudio económico consta de un reducido grupo de pacientes, creemos que las cifras obtenidas pueden ser más representativas de entornos como el nuestro y permitirnos cierta generalización para el resto del Sistema Nacional de Salud. La validez de tal extrapolación depende en gran medida de que los costes considerados de hospitalización sean relativamente homogéneos. En la tabla III puede verse nuestra estimación de costes comparadas con las procedentes de otras fuentes (2830). No se observan, en líneas generales, grandes diferencias. Nuestra apreciación puede considerarse incluso como conservadora, ya que se ha utilizado un GDR (131) con un coste económico inferior pero que fue el más utilizado en nuestro hospital para la codificación de procesos relacionados con la trombosis venosa. Ha sido señalada la alta variabilidad según las instituciones en los porcentajes de pacientes con ETEV que pueden ser tratados de forma ambulatoria y que puede oscilar entre el 18 y 91\% (31). En nuestro propio hospital, aunque condicionado por la reciente puesta en marcha de la Unidad de HADO, sólo 1 de cada 4 pacientes con ETEV fue tratado en régimen ambulatorio. Aun así, ello nos permite afirmar que si únicamente el $20 \%$ de los 14.000 pacientes ingresados cada año con el diagnóstico de TVP en los hospitales del SNS pudieran ser tratados ambulatoriamente podría ahorrarse el equivalente a 4,7 millones/año, suficientes para dotar cerca de 50 unidades de HADO semejantes a la nuestra.

En conclusión, en este trabajo se demuestra que la asistencia a pacientes con ETEV, aun aquellos con avanzada edad, importante comorbilidad y presencia de EP, puede realizarse de forma segura, eficaz y con notable ahorro de costes, mediante una unidad de HADO.

TABLA III

FUENTES DE DATOS DE COSTES HOSPITALARIOS RELACIONADOS CON LA ENFERMEDAD TROMBOEMBÓLICA VENOSA (EN EUROS)

\begin{tabular}{lcccc}
\hline Fuente (ref.) & Base Soykos, 2001 (28) & SNS referencia estatal, 1999 (29) & Rocha, 2002 (30) & H. Meixoeiro, 2002 \\
\hline TVP & $3133(2422-3840)$ (GRD128) & 1713 (GRD131) & 3030 & 1773 \\
EP & $3542(3217-2868)$ (GRD 78) & 3281 (GRD 78) & 3306 \\
\hline
\end{tabular}




\section{Bibliografía}

1. Grupo de Trabajo para las recomendaciones sobre el tromboembolismo venoso de la SISET (Prisco D, coordinador). Recomendaciones para el diagnóstico, la profilaxis y la terapia del tromboembolismo venoso. Haematologica 2004; 89 (Supl. 1): p. 1.

2. Stein PD, Huang HL, Afzal A, Noor HA. Incidence of acute pulmonary embolism in a General Hospital. Relation to age, sex and race. Chest 1999; 116: 909-13.

3. Hanson PO, Welin L, Tibblin G, Eriksson H. Deep vein thrombosis and pulmonary embolism in the general population. Arch Intern Med 1997; 157: $1665-70$.

4. Silverstein MD, Heit JA, Mohr DN, Petterson TM, O'Fallon WN, Melton LJIII. Trends in the incidence of deep vein thromboses and pulmonary embolism: a 25-year population-based study. Arch Intern Med 1998; 158: 585-93.

5. Cushman M, Tsai AW, White RH, Heckbert SR, Rosamond WD, Enright P, et al. Deep vein thombosis and pulmonary embolism in two cohorts: The Longitudinal Investigation of Thomboembolism Etiology. Am J Med 2004; 117: 19-25.

6. Bates SM, Ginsberg JS. Treatment of deep-vein thrombosis. N Engl J Med 2004; 351: 268-77.

7. Heit J, Melton LJ, Lohse CM, Petterson TM, Silverstein MD, Mohr DN, et al. Incidence of venous thromboembolism in hospitalized patients vs. community residents. Mayo Clin Proc 2001; 76: 1102-10.

8. Montes Santiago J, Rey García G, Mediero Domínguez A. Tromboembolismo pulmonar en pacientes médicos. Aproximación a los costes hospitalarios y tendencias evolutivas en España. An Med Interna (Madrid) 2004; 21: 326-330.

9. Warner GT, Perry CM, Ferguson JT. Enoxaparin in the prevention of tromboembolic disease in medical patients. Am J Cardiovasc Drugs 2001; 1: 477-82.

10. Gabriel Botella F. Reflexiones sobre la enfermedad tromboembólica venosa. An Med Interna (Madrid) 2003; 20: 447-450.

11. Soto-Álvarez J, González Vilchez J. Análisis costo-beneficio del uso de espironolactona en el tratamiento de la insuficiencia cardiaca crónica. Rev Esp Cardiol 2001; 54: 173-8.

12. Gould MK, Dembitzer AD, Sanders GD, Garber AM. Low-molecular weight heparins compared with unfractionated heparin for treatment of acute deep venous thrombosis. A cost-effectiveness analysis. Ann Intern Med 1999; 130: 789-799.

13. Rocha E, Martínez-González MA, Montes R, Panizo C. Do the low molecular weight heparins improve efficacy and safety of the treatment of deep venous thrombosis? A meta-analysis. Haematologica 2000; 85: 935-42.

14. Schraibman IG, Milne AA, Royle EM. Home versus in-patient treatment for deep vein thrombosis (Cochrane Review). In: The Cochrane Library, Issue 1, 2004. Chichester, UK: John Wiley \& Sons, Ltd.

15. Van den Belt AGM, Prins MH, Lensing AWA, Castro AA, Clark OAC, Atallah AN, et al. Fixed dose subcutaneous low molecular weight heparins versus adjusted dose unfractionated heparin for venous thromboembolism (Cochrane Review). In: The Cochrane Library, Issue 2, 2004. Chichester, UK: John Wiley \& Sons, Ltd.

16. Segal JB, Bolger DT, Jenckes MW, Krishnam JA, Steiff MB, Eng J, et al. Outpatient therapy with low molecular weight heparin for the treatment of venous thromboembolism: a review of efficacy, safety and costs. Am J Med 2003; 115: 298-308.
17. Schwarz T, Schmidt B, Höhlein U, Beyer J, Schröeder HE, Schellong SM. Eligibility for home treatment of deep vein thrombosis: prospective study. BMJ 2001; 322: 1212-13.

18. Grau E, Tenias JM, Real E, Medrano J, Ferrer R, Pastor E, et al. Home treatment of deep venous thrombosis with low molecular weight heparin: long term incidence of recurrent venous thromboembolism. Am J Hematol 2001; 67: 10-14.

19. Quinlan DJ, McQuillan A, Eikelboom JW. Low-molecular-weight heparin compared with intravenous unfractionated heparin for the treatment of pulmonary embolism. Ann Intern Med 2004; 140: 175-183.

20. Harrison L, McGinnis J, Crowther M, Ginsberg J, Hirsh J. Assesment of outpatient treatment of deep venous trombosis with low molecular weight heparin. Arch Intern Med 1998;158: 2001-3.

21. O’Brien B, Levine M, Willan A, Goeree R, Haley S, Blackhouse G, et al. Economic evaluation of outpatient treatment with low- molecular weight heparin for proximal vein thrombosis. Arch Intern Med 1999; 159: 2298-304.

22. Dunn AS, Schechter C, Gotlin A, Vomvolakis D, Jacobs E, Sacks HS, et al. Outpatient treatment of deep venous thombosis in diverse innercity patients. Am J Med 2001; 110: 458-462.

23. Instituto Nacional de Estadística. Encuesta de Morbilidad Hospitalaria. (Disponible en http: //www.ine.es/inebase/cgi/axi, consultado el 29/12/2003).

24. Martín-Carbonero L, Salgado X, Pedrajas JM, Armengol JG, Jiménez Rodríguez-Madridejos R, Fernández-Cruz A. Evolución a corto y largo plazo de la trombosis venosa profunda, tratada por una Unidad de Hospitalización a Domicilio. Rev Clin Esp 2002; 202: 430-4.

25. Suárez Álvarez CG, García Cañete J, Herrero Mendoza MD, Bellver Álvarez TM, Arboiro Pinel R. Tratamiento en domicilio de trombosis venosa profunda con heparinas de bajo peso molecular. Experiencia de un año. An Med Interna (Madrid) 2003; 20: 134-6.

26. Rocha Hernando E, Lecumberri Villamediana R, Feliú Sánchez J. Tratamiento del tromboembolismo venoso. En: Manejo práctico y pautas de actuación en la enferemedad tromboembólica venosa. Rocha Hernando E, Martínez Brotons F, Monreal Bosch M, eds. Acción Médica: Madrid, 2004; p. 123-140.

27. Cosín-Aguilar J. Análisis económico y de coste-beneficio en los tratamiento en cardiología. Enfoque en insuficiencia cardiaca. Rev Esp Cardiol 2001; 54: 136-8.

28. Brosa Riestra M, Rubio-Terrés C, Nadipell V, Casado Collado A, Martínez Brotons F. Análisis coste-efectividad de enoxaparina en la profilaxis de la enfermedad tromboembólica venosa en pacientes sometidos a cirugía mayor ortopédica. 1. Farm Hosp (Madrid) 2003; 27: 210-8.

29. Sistema Nacional de Salud. Año 1999. Explotación de bases del CMBD. Estadísticos de referencia estatal de los sistemas de agrupación de registros de pacientes (G.D.R.). Ministerio de Sanidad y Consumo, Subdirección General de Análisis Económico y Estadística; Madrid 2002, pp. 201 (GDR 78) y p. 250 (GDR 131).

30. Rocha E, Gómez-Outes A, Martínez-González J, Kakkar VV. Costeefectividad de bemiparina en el tratamiento agudo y alargo plazo de la trombosis venosa profunda. Bemiparina Proceeding books. $18^{\circ}$ Congreso Internacional Trombosis. Ljubljana, (Slovenia) 20-24 junio 2004, pp. 7-8.

31. Wells PS. Outpatient treatment of patients with deep-vein thrombosis or pulmonary embolism. Curr Opin Pulm Med 2001; 7: 360-4. 